\title{
A Nyquist Filter based Technique to Reduce PAPR in OFDM- MIMO Communication System
}

\author{
P. Chauhan ${ }^{1}$, P. Upadhyay ${ }^{2}$ \\ ${ }^{1}$ (Electronics and Communication, Maharishi Markandeshwar University Solan, India) \\ ${ }^{2}$ (Electronics and Communication, Maharishi Markandeshwar University solan, India)
}

\begin{abstract}
This paper describes the nyquist filter based technique to reduce the peak to average power ratio (PAPR) in multiple input multiple output Orthogonal Frequency Division Multiplication (MIMO-OFDM) systems. OFDM-MIMO system faces one of the main problems regarding PAPR of a transmitted signal. In present paper a nyquist filter along with conventional selected mapping (SLM) and alternative multiseqence (AMS) techniques are used for reducing the PAPR. The nyquist filter reduces the peak power of the transmitted signal and this may cause the reduction in PAPR. The simulated results are compared with conventional SLM and AMS techniques. Proposed nyquist filter based technique shows the lesser PAPR than the conventional SLM and AMS techniques. Coding and simulation has been done in Matlab 7.6.
\end{abstract}

Keywords: AMS, BER, MIMO, Nyquist Filter, OFDM, PAPR, SLM.

\section{Introduction}

Multiple-input multiple output orthogonal frequency division multiplexing (MIMO-OFDM) system have drawn significant interests due to its potential in achieving high data rate and providing reliable performance of diversity and spatial multiplexing. However, MIMO-OFDM system suffers from high peak-toaverage power ratio (PAPR) [1]. In MIMO-OFDM wireless system, independent OFDM signals are simultaneously transmitted from multiple-transmit antennas to multiple-receive antennas. Due to these multiple signals, PAPR problem occurs [2]. Recently, various algorithms of PAPR reduction have been proposed for OFDM system. There is a novel sub block linear combination scheme for peak-to-average power ratio reduction in OFDM system [3]. The key idea two novel methods is to divide an OFDM block into several subblocks, and iteratively generate the linear combination of these subblocks with low PAPR. One efficient technique for PAPR reduction is using low complexity partial transmitted sequence to construct of OFDM signals without side information[4]. In this scheme at the receiver, by utilizing the natural diversity of phase constellation for different blocks, the detector can successfully recover the original signal without side information. A nonlinear distributed companding scheme efficiently reduces PAPR with low Bit Error Rate. Therefore in [5] the novel scheme that transforms the OFDM signals into a trapezium distribution. The uniformly distributed companding scheme is a special case for PAPR reduction.

In [6] a constellation reshaping method to reduce the PAPR of OFDM signals without side information. A reshaped quadrature amplitude modulation (R-QAM) constellation is generated, then, the inherent diversity of the R-QAM constellation with different phase rotation factors could be exploited at the receiver. Clipping and filtering also effects the PAPR , crest factor and the power spectral density of the signal. However, clipping time domain signals causes out-of-band spectral regrowth and in band distortion. But the iterative clipping and filtering (ICF) procedure may be the simplest technique to approach a specified PAPR threshold in the processed OFDM symbols [7-8]. In [9] a PAPR analysis reduction of space-time block-coded (STBC) MIMOOFDM system for a $4 \mathrm{G}$ networks has been done. In [10] a modified selective mapping (SLM) technique for PAPR reduction of coded OFDM signal. In this technique, the phase sequence, this is used to lower the PAPR of the data block, in the check symbols of the coded OFDM data block. Both PAPR reductions from the SLM technique as well as error performance improvement from the channel coding with no loss in data rate from the transmission of side information. In [10-11] there is review and analysis different OFDM PAPR reduction techniques are given which is based on computational complexity, bandwidth expansion, spectral spillage and performance. These factors play an important role and effects PAPR of the signal. In another technique PAPR can be reduced by using precoding technique [12]. In this technique the precoding has been done at the transmitter end.

In [13] interleaving method is used for PAPR reduction. This method is also termed as adaptive symbol selection method .Multiple OFDM symbols are created by bit interleaving of input sequences and then the symbol with the lowest PAPR is selected at the receiver. Adaptive flipping of parity bits can also effect the PAPR of the OFDM signal [14]. In this method the PAPR ratio of the turbo coded OFDM signal is reduced with adaptive flipping of the phase of parity carriers. Dummy sequence insertion is also a PAPR reduction method shown in [15]. In this method the complementary sequence and the combination of the correlation sequence 
belong to the dummy sequence. Flipping technique can be combined into dummy sequence insertion method to effectively lower the PAPR reduction.

In this paper selected mapping and alternative multiseqence schemes along with nyquist filter has been used for reducing the PAPR of MIMO-OFDM based signals. Simulation results show that the proposed nyquist filter based technique have lesser PAPR than the conventional SLM and AMS Techniques.

The paper is organized as follows: Section 2 discuses about PAPR problem in MIMI OFDM system. Section 3 describes about conventional PAPR reduction techniques. In section 4 a discussion about proposed nyquist filter based technique is given. Section 5 discussed about simulation results and discussions. Finally section 6 concludes the paper.

\section{PAPR problem in MIMO-OFDM Systems}

Orthogonal frequency devision multiplexing (OFDM) is a multi-carrier modulation technique. OFDM technique provides the reasonable multipath delay spread tolerance, power efficiency, high spectral efficiency and immunity to the frequency selective fading channels. To modify the transmission capacity and quality, multiple input multiple output (MIMO) technique is used with OFDM for transmission purpose. High peak to average power ratio is one of the main problems with OFDM communication.

The major backlog of MIMO-OFDM system is that the signals transmitted on different antennas shows high peak-to-average power ratio.

PAPR is the challenging field of the MIMO-OFDM systems. In general, the PAPR of the MIMOOFDM signals at each antenna is defined as the ratio between the maximum power and the average power for the envelope of a baseband complex signal $\tilde{s}(t)$ i.e.

$$
\operatorname{PAPR}\{\tilde{s}(t)\}=\frac{\max |\tilde{s}(t)|^{2}}{\varepsilon|\tilde{s}(t)|^{2}}
$$

Let us denote the data block of length $\mathrm{N}$ as a vector $\mathrm{X}=\left[X_{0}, X_{1}, \ldots \ldots \ldots \ldots, X_{N-1}\right]^{\mathrm{T}}$ where $\mathrm{N}$ is equal to the number of subcarriers and $(\cdot)^{\mathrm{T}}$ denotes transpose. The duration of a data symbol $\mathrm{X}_{\mathrm{m}}$ in $\mathrm{X}$ is $\mathrm{T}$. Each data symbol in $\mathrm{X}$ modulates one of a set of subcarriers, $\left\{\mathrm{f}_{\mathrm{m}}, \mathrm{m}=0,1, \ldots \ldots \ldots, \mathrm{N}-1\right\}$. The $\mathrm{N}$ subcarriers are chosen to be orthogonal. That is, $\mathrm{f}_{\mathrm{m}}=\mathrm{m} \Delta \mathrm{f}$, where $\Delta \mathrm{f}=1 / \mathrm{NT}$ and NT is the time duration of an OFDM data block. The complex envelop of the transmitted OFDM signal is given by

$$
\mathrm{x}(\mathrm{t})=\frac{1}{\sqrt{\mathrm{N}}} \sum_{\mathrm{m}=0}^{\mathrm{N}-1} \mathrm{X}_{\mathrm{m}} \mathrm{e}^{\mathrm{j} 2 \pi \mathrm{f}_{\mathrm{m}} \mathrm{t}} \quad, 0 \leq t<\mathrm{NT}
$$

Therefore the PAPR of transmitted signal is defined as

$$
\mathrm{PAPR}=\frac{\max _{0} \leq t \in N T|X(t)|^{2}}{\frac{1}{N T} \cdot \int_{0}^{N T}|X(t)|^{2} d t}
$$

\subsection{Selected Mapping (SLM) Technique}

\section{Conventional SLM and AMS Techniques used for PAPR Reduction.}

The basic idea of this technique is first generate a number of alternative OFDM signals from the original data block and then transmit the OFDM signal having minimum PAPR. But data rate loss and complexity at the transmitter side are two basic disadvantages for this technique.

This is an effective and distortion less technique used for the PAPR reduction in OFDM. The name of this technique indicates that one sequence has to be selected out of a number of sequences. According to the concept of discrete time OFDM transmission we should make a data block considering $\mathrm{N}$ number of symbols from the constellation plot where $\mathrm{N}$ is the number of subcarriers to be used. Then using that data block $\mathrm{U}$ number of independent candidate vectors are to be generated with the multiplication of independent phase vectors. Let us consider $\mathrm{X}$ is the data block with $\mathrm{X}(\mathrm{k})$ as the mapped sub symbol (i.e. the symbol from the constellation). Where $\mathrm{k}=\{0,1,2, \ldots \ldots \ldots . . \mathrm{N}-1\}$. Let the $\mathrm{u}^{\text {th }}$ phase vector is denoted as $\mathrm{B}^{(\mathrm{u})}$, where $\mathrm{u}=\{1,2$, ..U $\}$. The $\mathrm{u}^{\text {th }}$ candidate vector that is generated by the multiplication of data block with the phase vector is denoted as $\mathrm{X}^{(\mathrm{u})}$. So we can write the equation to get the $\mathrm{k}^{\text {th }}$ element of $\mathrm{u}^{\text {th }}$ candidate vector as:

$$
X^{(w)}(k)=X(k) B^{(w)}(k)
$$

Then by doing IFFT operation to each candidate vector we will obtain U number of alternative OFDM signals, so the $\mathrm{n}^{\mathrm{th}}$ symbol of $\mathrm{u}^{\text {th }}$ alternative OFDM signal can be written mathematically as:

$$
X^{(w)}(n)=\frac{1}{\sqrt{N}} \sum_{k=0}^{N-1} X^{(W)}(k) e^{j\left(\frac{2 \pi n k}{N}\right)}
$$

So out of the U number of alternative OFDM signals the signal having minimum PAPR is to be selected for transmission. So in this technique for generation of alternative OFDM symbols the independent phase vectors 
has to generate. This selected OFDM signal at transmitter side has to be detected at the receiver. So the receiver must have the information about the perfect phase vector that has been multiplied to generate that selected OFDM signal. The block diagram of SLM technique is given as in fig 1.

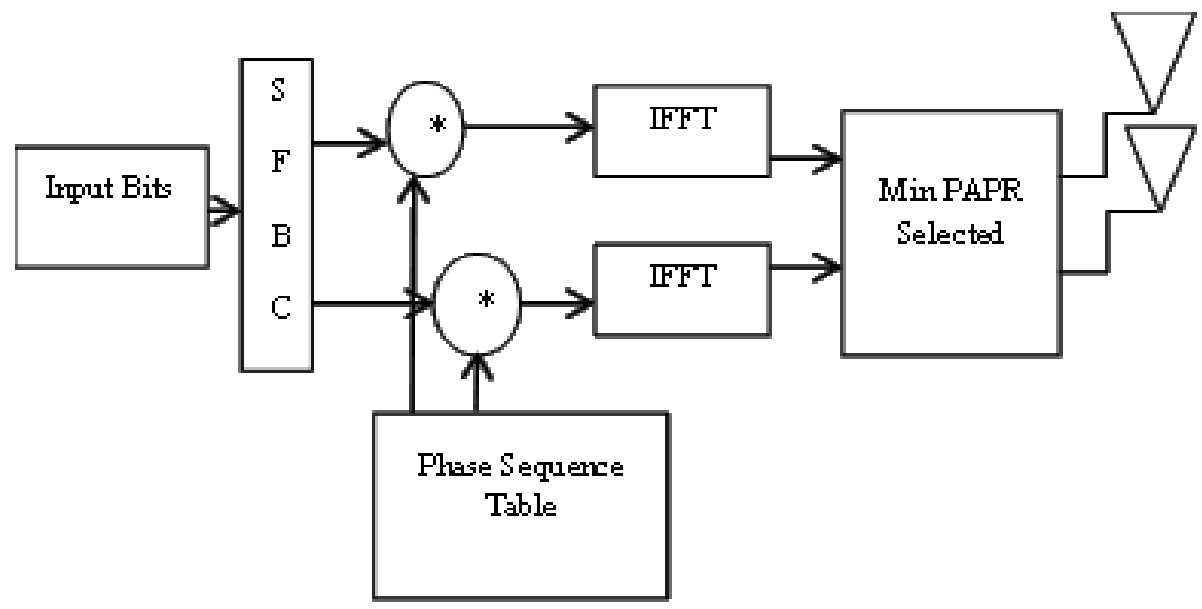

Fig. 1 Block diagram of selected mapping technique.

\subsection{Alternative Multisequence Scheme (AMS)}

Alternative Multisequence (AMS) scheme is used for reducing the PAPR of MIMO-OFDM signals. In AMS method, original data sequences at two antennas are divided into several couples of sub blocks, and each couple of sub blocks multiplies by different factors to get different pair of sub blocks. Then, the new sub blocks obtained are combined to get AMSs, which keep the structure. Finally, the couple of alternative sequences with the smallest PAPR is chosen to be transmitted. The block diagram of the AMS technique is given in fig 2.

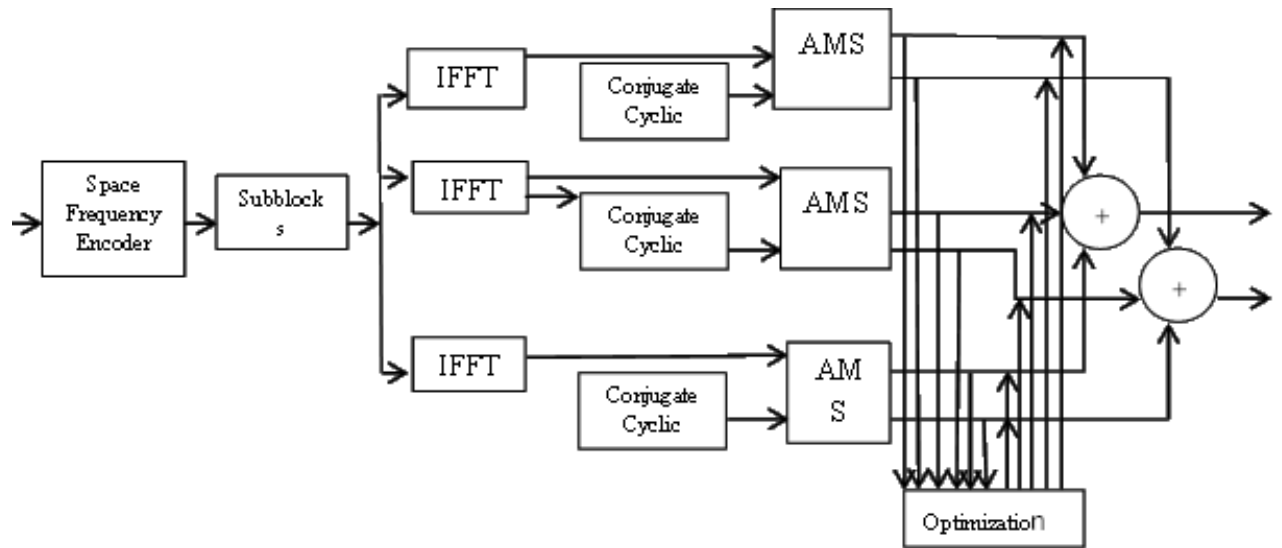

Fig. 2 Block diagram of Alternative Multisequence Technique.

\section{Proposed Nyquist Filter Based PAPR Reduction Technique}

Selected Mapping Technique \& Alternative Multisequence Scheme both are used to reduce PAPR of MIMO-OFDM systems. In the proposed work SLM and AMS along with nyquist filter are used for reducing the PAPR. Nyquist filters is used in various applications such as interpolation filter, reconstruction filter bank, nonuniform sampling and many more. Except for one point the impulse response at nyquist rate should be zerocrossing. It is used by using either IIR or FIR filters. With exact linear phase the design of FIR Nyquist can be made. Still a higher order FIR Filter is required for getting the specification of sharp magnitude and there is a delay results. This is due to the reason because the half of filter order for exact linear-phase FIR filter is equal to group delay. A low delay is required in some cases of real-time signal processing. Transfer function of FIR filter shown in (6).

$$
H(z)=\sum_{n=0}^{N} h_{n} z^{-n}
$$

Where $\mathrm{H}(\mathrm{z})$ is transfer function of FIR digital filter of order $\mathrm{N}$. The block diagram of proposed nyquist filter based scheme is given in fig 3 . 


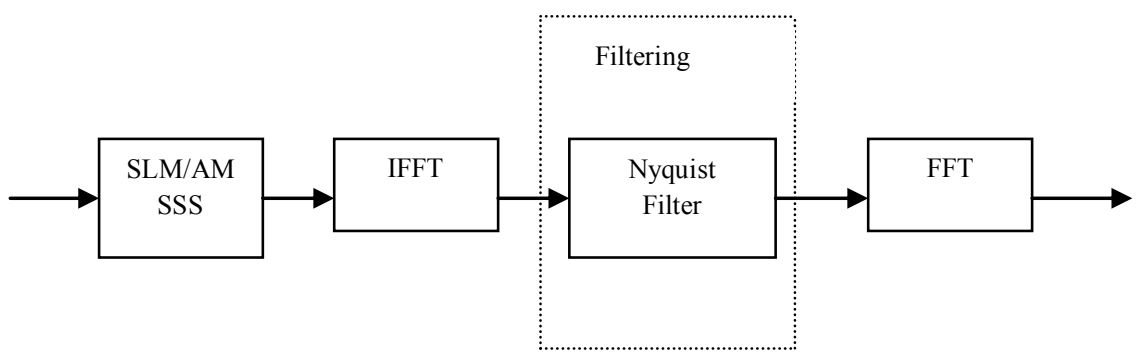

Fig. 3 Block Diagram of Proposed Nyquist Filter based Technique.

\subsection{Nyquist pulse shaping}

The Nyquist pulse is the signal pulse required to communicate over band-limited channels with no ISI. However, only a few discuss their digital version counterpart that is generally used in practice. For a pulse duration of L samples the digital pulse-shaping filter should satisfy the so-called Nyquist condition for zero ISI, i.e. the impulse response such that

$$
h_{N y q}[k L]=0
$$

A frequently used class of filters verifying the Nyquist criteria is the raised cosine RC filters. The frequency response of an RC filter in the continuous-time domain is shown in (8)

$H_{R C}(j \omega)=\left\{\begin{array}{l}T_{S Y M B} \\ \frac{T_{S Y M B}}{2} \\ 0\end{array}\left\{1+\cos \left[\frac{T_{S Y M B}}{2 \alpha}\left(|\omega|-\frac{(1-\alpha) \pi}{T_{S Y M B}}\right)\right]\right.\right.$

where $T_{\text {SYMB }}$ is the symbol's duration time period and $\alpha$ is the filter roll-off which specifies the excess bandwidth with respect to $1=\left(2 \mathrm{~T}_{\mathrm{SYMB}}\right)$ (the minimum bandwidth required, according to Nyquist theory to transmit a sequence at rate $\left.1=\mathrm{T}_{\mathrm{SYMB}}\right)$.

In order to maximize the signal-to-noise ratio (SNR) at the output of the receiver's front-end the receiver filter must be matched to the transmitting shaping filter. Hence, the overall RC spectral characteristic is usually split evenly between the filters used at the transmitter and receiver, i.e. both having a spectra of magnitude $\left|\mathrm{H}_{\mathrm{RRC}}(\mathrm{jw})\right|=\sqrt{\left|H_{R C}(j \omega)\right|}$, which is why this filter is called root-raised cosine.

The impulse response of a digital RRC is obtained by sampling the continuous time impulse response $\mathrm{H}_{\mathrm{RRC}}(\mathrm{t})=\mathrm{f}^{1}\left\{\mathrm{H}_{\mathrm{RRC}}(\mathrm{jw})\right\}$, at a rate $\mathrm{f}_{\mathrm{SMPL}}=\mathrm{L} / \mathrm{T}_{\mathrm{SYMB}}$, which gives in $(9)$

$h_{R R C}[n]=\frac{4 \alpha n^{2} \cos \left[\frac{\pi n(1+\alpha)}{L}\right]+L \sin \left[\frac{\pi n(1+\alpha)}{L}\right]}{\left[1-\left(4 \alpha \frac{n}{L}\right)\right] \pi n \sqrt{L}}$

Which was scaled, to ensure $\sum_{n}|h(n)|^{2}=1$. The autocorrelation of RRC impulse response, i.e. $h_{R R C}[n] * h_{R R C}^{*}[-n]$, is nyquist filter.

The oversampling factor $\mathrm{L} € \mathbf{N}$ determines the up-sampling factor that precedes the pulse shaping filter. According to the Nyquist sampling theorem in order to avoid aliasing we must guarantee that

$\mathrm{f}_{\mathrm{SMPL}}[n] \geq \frac{2}{T_{S M B L}}(1+\alpha)$

In practice, the use of a higher up-sampling factor imposes fewer restrictions on the design of the DAC's reconstruction filter, allowing a considerable reduction in its order and so lowering its cost . Therefore, it is usual to operate the digital RRC filter at 4 or 8 samples per symbol, i.e. to set $\mathrm{L}=4$ or 8 .

To limit the impulse response $h_{R R C}[\mathrm{n}]$ to a finite duration, $h_{R R C}[\mathrm{n}]$ is usually truncated by applying a rectangular window that spreads over $2 \mathrm{~N}+1$ symbol. The parameter $\mathrm{N}$ should be large enough to guarantee almost zero ISI. To solve this problem and also the fact that the side-lobes in the spectrum of the finite duration 
$h_{R R C}[n]$ are quite high (rectangular windowing), other windows are also used or, alternatively, filter coefficients are optimized.

Consider the generic block diagram of a typical SC transmitter. It is our intention to prove that the PAPR of a single carrier band limited signal $x[n]$ is shown in (11)

$\mathrm{PAPR}_{\mathrm{X}[\mathrm{N}]}=\mathrm{PAPR}_{\text {Const }}+\mathrm{PAPR}_{\mathrm{RRC}}$

Symbols at the output of the demodulator are assumed to be independent and identically distributed random variables .As shown before; the transmitted average power is given by

$\sigma_{S=E}^{2}(|S[n]|)^{2}=\frac{1}{M} \sum_{i=1}^{M}|S|_{i}^{2}=\varepsilon_{s}$

PAPR is expressed as shown in equation (13)

$\operatorname{PAPR}_{s[n]}=10 \log _{10}\left(\frac{\max \left|s[n]^{2}\right|}{\sigma_{s}^{2}}\right)(\mathrm{dB})$

In order to abtain the PAPR of signal $\mathrm{x}[n]$, we just have to evaluate maximum value of $\mathrm{x}[n]$ as shown in equation (14)

$\operatorname{Max}|\mathrm{x}[\mathrm{n}]|=\max \left|\sum_{k} h[k] s_{n}[n-k]\right|$

Since the transmitter system is multirate the pulse-shaping can be performed at symbol rate according tp polyphase decomposition, as presented. Denoting the output signal of each polyphase filter, we get

$$
\begin{aligned}
& \max _{n}|x[n]|=\max _{i=0,1, \ldots \ldots, L-1}\left|y_{i}[n]\right| \\
& \quad=\max _{i=0,1, \ldots, L-1}\left|\sum_{k} h\right|[k L+i] S_{u}[n-k] \mid
\end{aligned}
$$

Which, according to algebra properties, can be upper bounded as

$$
\begin{aligned}
\max _{n}|x(n)|^{2} & \leq \max _{i=0, \ldots, L-1} \sum_{k}\left|h[k L+i] s_{u}[n-k]\right| \\
& \leq \max _{n}|s[n]| \times \max _{i=0, \ldots, L-1} \sum_{k}|h[k L+i]|
\end{aligned}
$$

The PAPR of $\mathrm{x}[n]$ can thus be computed as

$$
\begin{aligned}
\operatorname{PAPR}_{x[n]} & =10 \log _{10}\left(\frac{\max [x[n]]^{2}}{\sigma_{x}^{2}}\right) \\
& =10 \log _{10}\left(\frac{\max _{n}|s[n]| \times \max _{i=0, \ldots, L-1} \sum_{k} \mid h[k z+i||}{\frac{\sigma_{s}^{2}}{L} \sum_{n=-\infty}^{\infty}|h[n]|^{2}}\right)
\end{aligned}
$$

Which can be written as

$$
\operatorname{PAPR}_{x[n]}=10 \log _{10}\left(\frac{\max _{n}|s[n]|}{\sigma_{s}^{2}}\right)+10 \log _{10}\left(\frac{\max _{i=0, \ldots, L-1} \sum_{k}|h[k L+i]|}{\frac{1}{L} \sum_{n=-\infty}^{\infty}|h[n]|^{2}}\right)
$$

Here the nyquist filter reduces the peak power of the transmitted signal and this causes the reduction in PAPR.

\section{Result and Discussion}

This work considers different experiments to analyze the performance of PAPR in MIMO-OFDM using nquist filter along with the AMS and SLM scheme. The simulation has been carried out for 4-QAM and 16-QAM system. At the transmitter the sequence of information bits is fed to a block encoder that maps a block 
of bits into signal points selected from a signal constellation consisting of signal points. The bit interval block is used to logically reorder the data. A mapper block is used in the QAM which is followed by the serial to parallel converter. The process of mapping the information bits onto the signal constellation plays a fundamental role in determining the properties of the modulation. Bit Error Rate and PAPR has been calculated for proposed nyquist filter based technique for both 4-QAM and 16-QAM and results are compared with conventional SLM and AMS schemes.

Figures 4 and 5 shows the matlab simulated result for peak to average power ratio (PAPR) for 4-QAM for conventional AMS and SLM techniques and proposed nyquist filter based AMS and SLM techniques respectively.

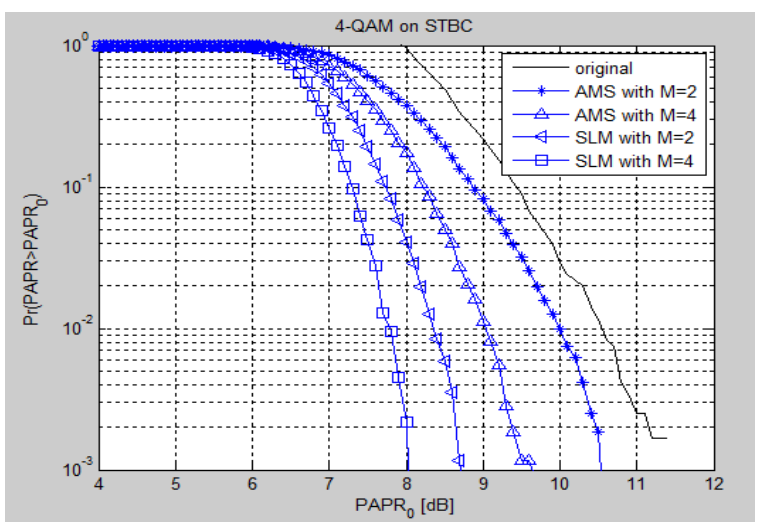

Fig. 4 PAPR curves of Conventional SLM and AMS Technique for 4-QAM.

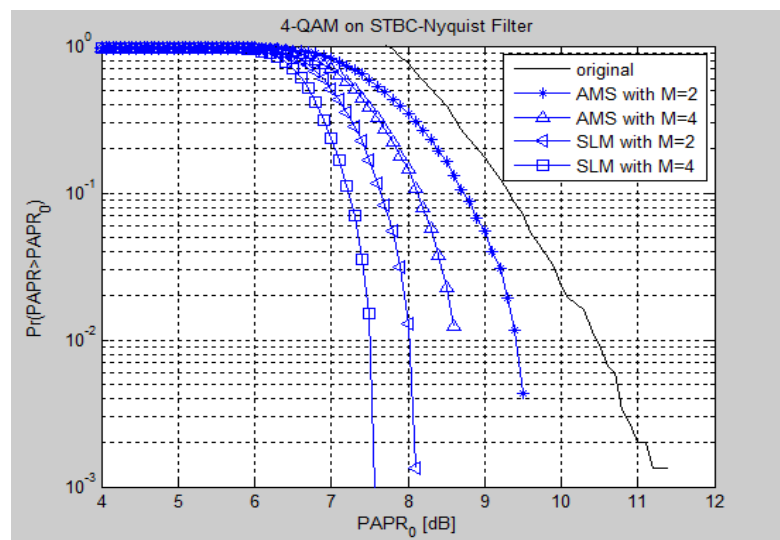

Fig. 5 PAPR curves of Proposed Nyquist Filter based Techniques for 4-QAM.

From the Figure 5 it is evident that the proposed nyquist filter based technique has lesser value of PAPR than the conventional SLM and AMS techniques.

Similarly Figures 6 and 7 shows the matlab simulated result for peak to average power ratio (PAPR) for 16-QAM for conventional AMS and SLM techniques and proposed nyquist filter based AMS and SLM techniques respectively.

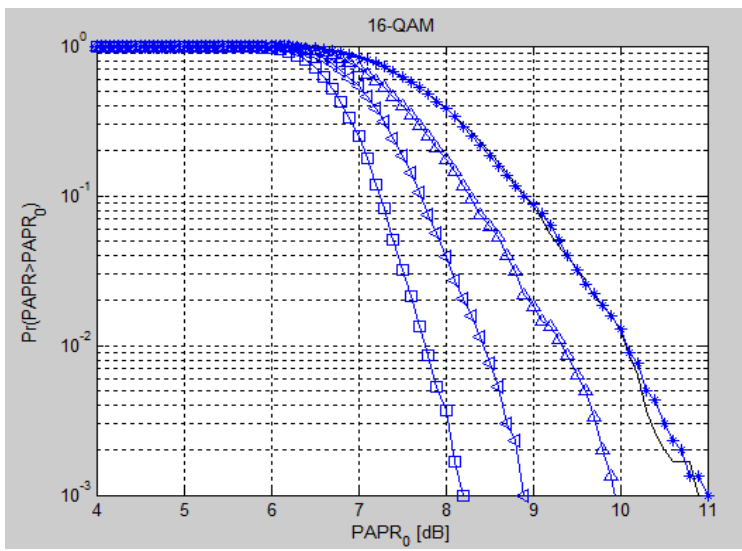

Fig. 6 PAPR curves of Conventional SLM and AMS Technique for 16-QAM. 
Following graph implies that the PAPR for 16-QAM for schemes SLM and AMS along with the Nquist filter is lower than the conversional schemes. Which can be seen in fig.7.

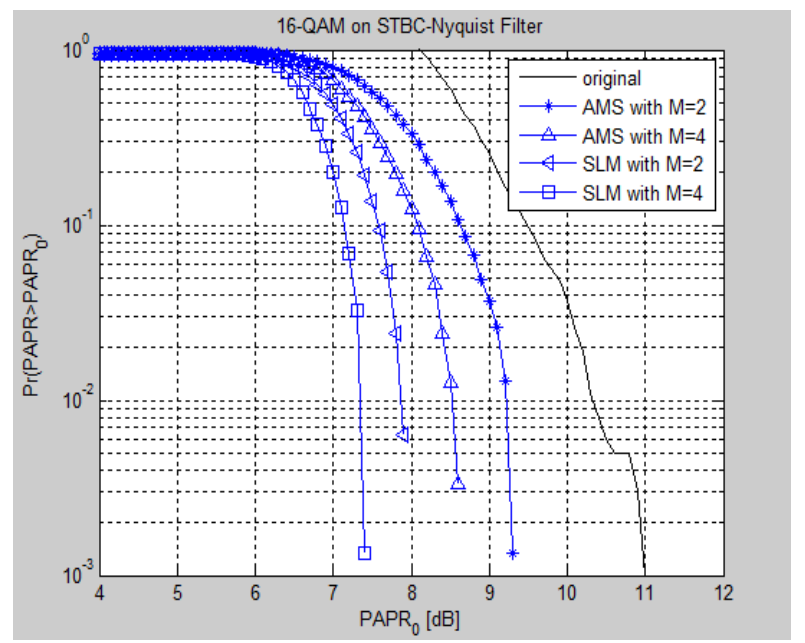

Fig. 7 PAPR curves for Proposed Nyquist Filter based Techniques for 16-QAM.

Figure 7 shows that proposed nyquist filter based technique having lesser value than the conventional SLM and AMS techniques.

Table 1. PAPR Comparison for Proposed and Conventional Techniques

\begin{tabular}{|c|c|c|c|c|c|c|c|c|}
\hline \multirow{3}{*}{$\begin{array}{l}\text { Technology } \\
\text { Used }\end{array}$} & \multicolumn{8}{|c|}{ Peak to average power ratio (PAPR) Values (dB) } \\
\hline & \multicolumn{2}{|c|}{$\begin{array}{l}\text { Conventional Selected } \\
\text { Mapping (SLM) [1] }\end{array}$} & \multicolumn{2}{|c|}{$\begin{array}{l}\text { Proposed Nyquist filter } \\
\text { based SLM Technique }\end{array}$} & \multicolumn{2}{|c|}{$\begin{array}{c}\text { Conventional } \\
\text { Alternative } \\
\text { Multisequence (AMS) } \\
{[2]}\end{array}$} & \multicolumn{2}{|c|}{$\begin{array}{l}\text { Proposed Nyquist filter } \\
\text { based AMS Technique }\end{array}$} \\
\hline & $\mathrm{M}=2$ & $M=4$ & $M=2$ & $M=4$ & $\mathrm{M}=2$ & $\mathrm{M}=4$ & $\mathrm{M}=2$ & $M=4$ \\
\hline 4-QAM & 8.7 & 8.0 & 8.1 & 7.5 & 10.5 & 9.8 & 9.0 & 9.5 \\
\hline 16-QAM & 9.0 & 8.3 & 8.0 & 7.4 & 11.0 & 10.0 & 9.3 & 8.6 \\
\hline
\end{tabular}

Table 1 shows the comparison between the reported values of PAPR and the proposed nyquist filter based technique. PAPR value for proposed nyquist filter based SLM technique is $8.1 \mathrm{~dB}$ and $7.5 \mathrm{~dB}$ for 4$\mathrm{QAM}$ at $\mathrm{M}=1$ and $\mathrm{M}=2$ respectively. Similarly, PAPR value for proposed nyquist filter based AMS technique is $9.0 \mathrm{~dB}$ and $9.5 \mathrm{~dB}$ for $16-\mathrm{QAM}$ at $\mathrm{M}=1$ and $\mathrm{M}=2$ respectively. The Table 1 it is clear that the proposed nyquist filter based technique has least value of PAPR for both 4-QAM and 16-QAM technology.

\section{Conclusion}

MIMO-OFDM systems are used in mobile communications systems where the higher data rate transmission can be achieved gainfully using the small bandwidth available. Here in this paper a nyquist filter is used along with AMS and SLM techniques for reducing the peak average power of the transmitted signal. Reduction in peak power reduces the PAPR value of the signal and enhances the quality of the signal. The simulated results for proposed nyquist filter based technique are compared with that of conventional SLM and AMS techniques reputed in the literature. The results for proposed technique shows the least value of PAPR that the conventional SLM and AMS technique. So it will be conclude that the proposed nyquist based technique provides the better solution for PAPR reduction. Although the complicity and hardware of the proposed system is increased in comparison to conventional techniques but lesser value of PAPR can shadow this drawback.

\section{References}

[1] T. Jiang, C. Ni and L. Guan, A Novel Phase Offset SLM Scheme for PAPR Reduction in Alamouti MIMO-OFGDM System Withiout Side Information, IEEE Signal processing letters,20(4), 2013, 383-386.

[2] T. Jiang and C. Li, Simple Alternative Multisequence for PAPR Reduction Without Side Information in SFBC MIMO-OFDM system, IEEE Transaction Vehicular Technology, 61(7), 2012, 3311-3315.

[3] H. Li, T. Jiang, A novel sub block linear combination scheme for peak to average power ratio reduction in OFDM system, IEEE Transaction on Braodcasting, 58(3), 2012, 360-369.

[4] L. Yang, K. K. Soo, S. Q. Li, and Y. M. Siu, PAPR Reduction using Low Complexity PTS to Construct of OFDM signals Without side information, IEEE Transaction on Broadcasting, 57(2), 2011, 284-290

[5] S.S. Jeng and J-M. Cheng, Efficient PAPR Reduction in OFDM system Based on a Companding Technique with Trapezium Distribution, IEEE Transaction on Braodcasting, 57(2), 2011, 291-298. 
[6] C. Li, T. Jiang, Y. Zhou and H. Li, A Novel Constellation Reshaping Method for PAPR Redduction of OFDM Signal, IEEE Transaction on Signal Processing, 59(6), 2011, 2710-2718.

[7] X. Li, L.J. Cimini, Effect of Clipping and Filtering on the Performance of OFDM, IEEE Communication Letters, 2(5), 1998, 131133.

[8] Y.C. Wang, Z.Q. Luo, Optimized Iterative Clipping and Filtering for PAPR Reduction of OFDM Signals, IEEE Transaction on Communication, 59(1), 2011, 33-37.

[9] S. H. Wang and C. P. Li, A low - complexity PAPR reduction scheme for SFBC MIMO-OFDM system, IEEE Signal Process. Lett., 16(11), 2009, 941-944.

[10] T. Jiang and Y. Wu, An Overview: Peak-to-Average Power RatioReduction Techniques for OFDM signal, IEEE Transaction on Braodcasting, 54(2), 2008, 257-268.

[11] M. Khan, S, Iqbal and W. Asghar, A review paper on: The PAPR Analyses of Orthogonal Frequency Division Multiplexing, International journal of Mobile Network Communications \& Telematics ( IJMNCT), 4(1), 2014.

[12] S. B. Slimane, Reducing the peak-to-average power ratio of OFDM signals through precoding," IEEE Trans. Veh. Technol., 56(2), 2007, 686-695.

[13] Q. Zhang, F. Yu, and A. Nallanathan, Improving Achievable Rates In MPSK Amplify And Forward Relay Network Via Clipping, IEEE Transaction on Vehicular Technology, 59(7), 2010, 2133-2137.

[14] O. Muta, Y. Akaiwa, Weighting factor Estimation Method for Peak Power Reduction Based on Adaptive Flipping of Parity Bits in Turbo- Coded OFDM System, IEEE Transaction Vehicular Technology ,57(6), 2008, 3551-3562.

[15] H.-G. Ryu, J.-E. Lee and J.-S. Park, Dummy sequence insertion (DSI) for PAPR reduction in the OFDM communication system, IEEE Transaction on Vehicular Technology, 50(1), 2004, 89-94. 